\title{
Field Measurement Based PLS Model for Dynamic Rating of Overhead Lines in Wind Intensive Areas
}

\author{
S. Abdelkader, D. J. Morrow, J. Fu and S. Abbott \\ School of Electronics, Electrical Engineering \& Computer Science \\ Queen's University Belfast \\ Ashby Building, Stranmillis Road, Belfast, BT9 5AH (United Kingdom) \\ Phone:+44 28 90974142, e-mail: s.abdelkader@qub.ac.uk, dj.morrow@qub.ac.uk, jfu03@qub.ac.uk, \\ s.abbott01@qub.ac.uk
}

\begin{abstract}
This paper presents results of lab tests, field measurements and mathematical modelling for the Dynamic Rating (DR) of Over Head Transmission Line (OHTL) in a wind intensive area. In this paper, DR is done by developing a statistical line model. This model is based on Partial Least Squares (PLS) multi regression. DR provides extra capacity to the line, over the traditional seasonal static rating, which makes it possible to defer the need for reinforcement the existing network or building new lines.
\end{abstract}

\section{Key words}

Dynamic line rating, transmission line, wind generation integration, partial least squares regression.

\section{Introduction}

thermal rating of an OHTL is usually determined for the different seasons based on assumed worst weather conditions using either the IEEE [1] or the Cigre model [2]. The line rating is kept fixed for the whole season, which is known as static rating. Being based on worst weather conditions, static line rating ensures safety and security. However, due to the conservative assumptions, the line is underutilized most of the time. Therefore, Dynamic Line Rating (DLR) has been considered for a long time as a means of better utilization of the available assets and deferring the need to building new infrastructures. This has led to many research works and applications for DLR [3-10]. These works cover a wide range of methods including sag measurement, thermal models for the conductor ether by including the effect of all weather parameters, weather model (WM), or by using just the conductor temperature, when it is higher than the ambient temperature by 10 degrees or more, which is known as conductor temperature model (CTM). Determination of the line section with the highest temperature, the critical span, has also been considered in many research works and field studies [11].

The availability of new measurement technologies such as GPS and PMU has resulted in developing new methods for DLR. GPS measurements were used in [12] to measure conductor sag and hence determine its available rating. In [13], PMU measurements were used to determine line parameters, then the conductor temperature and the available additional capacity can be determined.

Wind power is the major renewable energy source in the UK and most of EU countries. Therefore, it is the expected major contributor in the fulfillment of the ambitious targets of integrating more power from renewable energy sources. Wind farms are usually installed in rural areas where a strong grid is not available. The available alternatives to accommodate the wind power are either build new lines to transmit the windgenerated power to load centers, or to up rate the existing lines by some way or another. Building a new transmission line requires more time than installing a wind farm. The cost of the rights of way and concerns about the environment are most likely to prohibit, or at least limit, building new lines. This gives DLR greater opportunity, especially in wind intensive areas; because when wind is generating power it is cooling the conductors and hence makes more transmission capacity available without the need of new infrastructures.

This paper presents a description of some activities within a project carried out at Queen's University Belfast (QUB) for developing a model for DLR. These include lab tests, field data logging and mathematical modeling. The parts describing the tests have been published in previous reports and papers [1416] by the project team. The main contribution of this paper is the description and validation of the DLR model.

The rest of the paper is organized as follows: Section II presents a description of the lab tests explaining the set up of each test, its purpose and the outcome of each test and how it helps in developing the DLR model. In section III, the field data logging system is described. Section IV presents the proposed PLS model for DLR with results of application to field measurements and comparison of the model predicted conductor temperature and the measured one. A conclusion is presented in section $\mathrm{V}$ followed by list of references. 


\section{Lab Tests}

Lab tests have been carried out to investigate conductor behavior in different controlled conditions. The main purpose is to gain more insight into the thermal behavior of the conductor in terms of dependence of conductor temperature on different parameters including current and wind speed. Thermal time constant of the conductor is an important output of these tests, as it helps deciding on the structure of the DLR model as will be explained later. Two sets of tests were carried out, still air test and wind tunnel tests. Full results of these tests are reported in [14]. The following two sections give a description of the experimental set up and the most important results of the two sets of tests. All tests are carried out using a segment of the Lynx $175 \mathrm{~mm}^{2}$ conductor. Length of the conductor used is determined by the distance between the two walls of the QUB high voltage lab, which is $4.1 \mathrm{~m}$ for the still air test; and only $1.5 \mathrm{~m}$ for the wind tunnel test.

\section{A. Still Air Test}

The schematic diagram of the still air test is shown in Fig.1 The line segment to be tested is supported by two brackets and connected to a special transformer which can supply up to $1600 \mathrm{~A}$ at a voltage of $3 \mathrm{~V}$ for one minute. The continuous rating of the transformer is 653A. Transformer output is regulated by a variac. Thermocouples are mounted on the conductor surface to measure the conductor surface temperature. A $9.8 \mathrm{~mm}$ hole was made in the conductor and a thermocouple was fixed in it to measure the conductor core temperature. The FMC sensor shown in the figure is the real time monitoring equipment used for continuous field measurement of conductor temperature. It has been connected here for the purpose of calibration. The thermocouples and the FMC temperature sensor have been attached to the conductor using a heat sink compound to ensure a good heat transfer between the conductor and the sensors. Fig 2 shows a photo of a FMC's temperature sensor attached to the conductor service. Fig. 3 shows a photo of the actual still air test rig set up within the high voltage lab at QUB.

The main purpose of this test is to compare the conductor behavior in still air conditions with those in the wind tunnel tests to determine the effect of wind on cooling the conductor and hence increasing its rating; also to calibrate the FMC sensor which is used for field measurement and online monitoring.

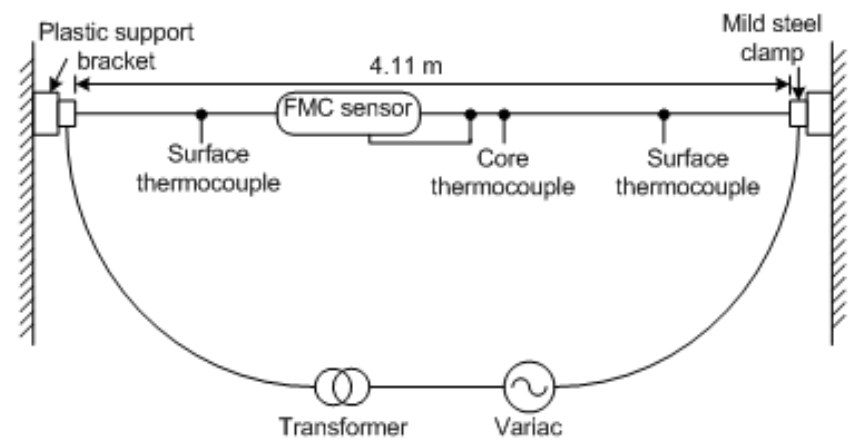

Fig. 1. Schematic diagram of the still air test

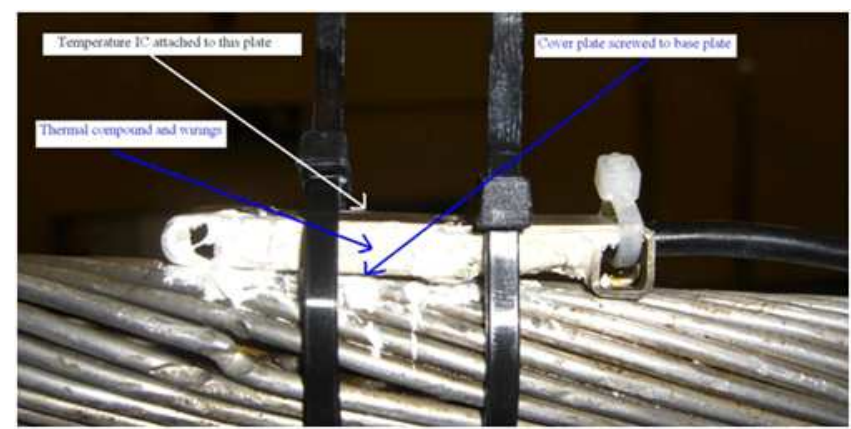

Fig. 2. Attaching FMC sensor to the conductor

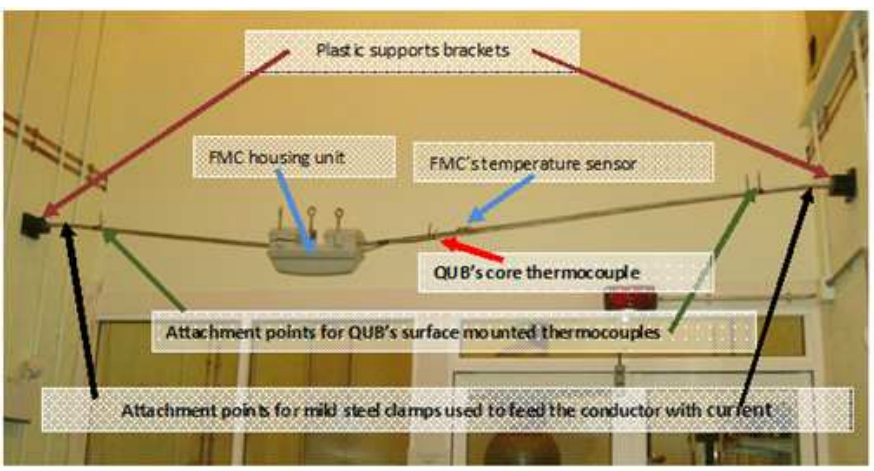

Fig. 3. Still air test rig set up at QUB HV Lab

The conductor temperature response to a 550 A step increase in current is shown in Fig. 4. It can be observed that the surface temperature is larger than the core temperature at the start of current step application. This is due to the skin effect that makes most of the current flow near the conductor surface. However, after some time, the core temperature becomes higher than the surface temperature. This is due to the fact that the heat generated at the conductor surface is dissipated to the surrounding air whereas the heat generated in the core has to pass through the conductor to the surface.

An important thing to notice also from Fig. 4 is the decrease in conductor current with time although both the variac and the transformer are set to supply a fixed voltage to the conductor. The increase in conductor resistance with the increase in its temperature is obviously the main reason for this decrease in current magnitude. The variation in conductor current is about $8 \%$, which means that the conductor impedance variation is $8.6 \%$ and the resistance variation would be even higher bearing in mind that the conductor reactance is independent on temperature. Such a large variation in conductor resistance cannot be tolerated in the transient modeling of the thermal behavior of the conductor to determine its DR; but it is not straight forward to accurately include it. This was the main motive for us to develop a model independent of conductor parameters to avoid inaccuracies resulting from assumptions regarding the conductor parameters. More details about this test can be found in [14].

\section{B. Wind Tunnel Tests}

The setup for this test is just like the still air test except that a shorter length of the line, about $1.5 \mathrm{~m}$, is mounted inside the wind tunnel due to space limitation. Three thermocouples are also attached to the conductor, two for measuring surface temperature and one for measuring the 
conductor core temperature. Power supply is the same used for the still air test. A laminar air flow, with controllable speed, is passed perpendicular to the conductor. Fig. 5 shows the wind tunnel test rig. A comprehensive description of this test and its outcomes are reported by the project team members [15]. In this paper some results of this test are presented to make it clear how the proposed model was developed and how its structure was guided by the results of these tests.

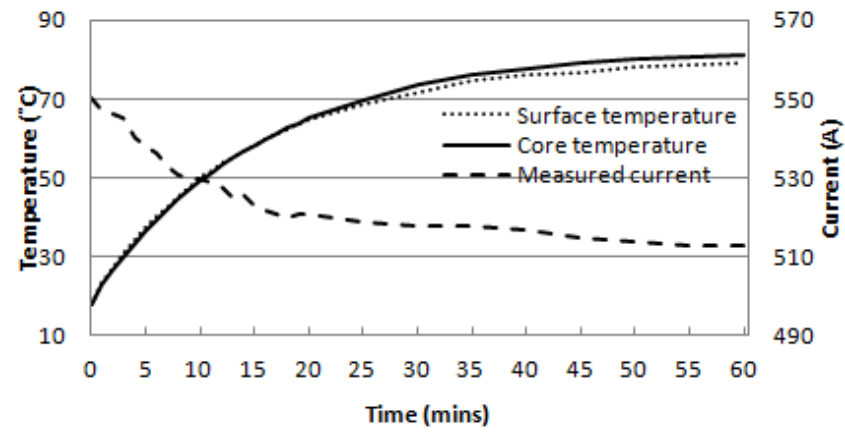

Fig. 4. Conductor temperature response to a step current

Fig. 6 depicts the time response of the conductor temperature to a step current of $550 \mathrm{~A}$ at different wind speeds. The figure clearly shows the effect of wind speed on the conductor temperature. The conductor temperature dropped from about 95 degrees at zero wind speed to slightly above 50 degrees at $1 \mathrm{~m} / \mathrm{s}$ wind speed and to less than 30 degrees for wind speed of $15 \mathrm{~m} / \mathrm{s}$. It is interesting to notice that the temperature rise time and hence the thermal time constant of the conductor is dependant on wind speed.

The figure reveals a very important feature of wind cooling that is the wind cooling effect on conductor temperature is more noticeable at lower wind speeds. As the wind speed gets higher the cooling effect reaches a kind of saturation where the increase in cooling gets less. The steady state conductor temperature is plotted against the wind speed in Fig. 7 both that measured from the wind tunnel test and an exponential fit to the measured data.

Lab tests have provided very helpful information, which helped to set guidelines for developing a model for the thermal behavior of the conductor. The proposed PLS model is a multivariate linear regression model. Of course, it is a static model based on statistical data obtained through measurements. Nonetheless, the quantities used as independent variables, the rate at which data is collected and the number historical data to be used are all chosen in a way that makes the model imitates the transient thermal behavior of the conductor.

Thermal time constant of the conductor is used as a guide of how should be the measurement rate of weather parameters. Time constants obtained from the wind tunnel test are listed in Table I, which shows that these time constants ranges from 7.6 down to 1.3 minutes for core temperature and slightly less values for the surface temperature. Therefore, the measurement step was chosen to be 5 minutes, almost 3 times the time constant at the average wind speed. This is to leave a sufficient time for conductor temperature to respond to variations taking place between the two measurements. To account for the probable situations of low wind speeds where the time constant is larger, 3 consecutive measurements covering time span of 15 minutes, twice the highest time constant, are used in the model as will be discussed later.

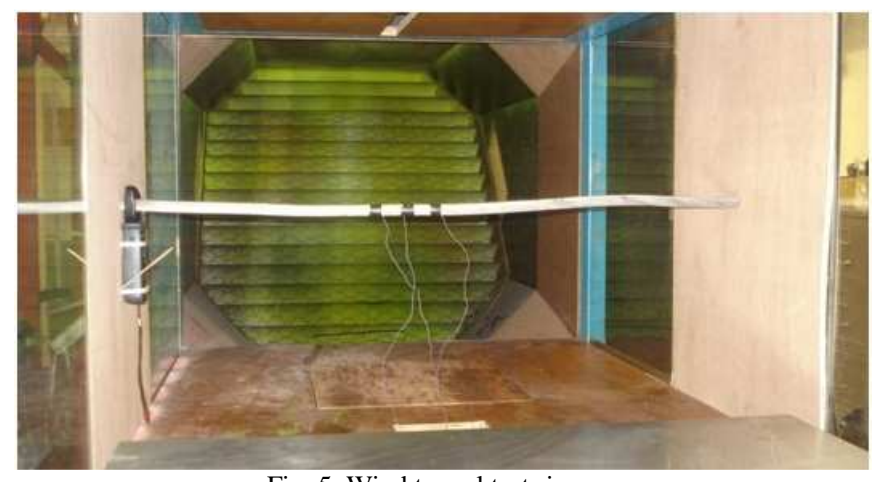

Fig. 5. Wind tunnel test rig

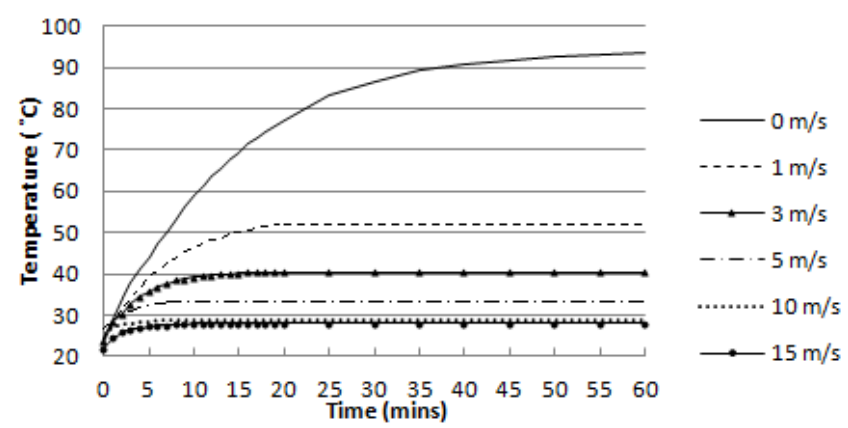

Fig. 6 Time variation of conductor temperature at different wind speeds

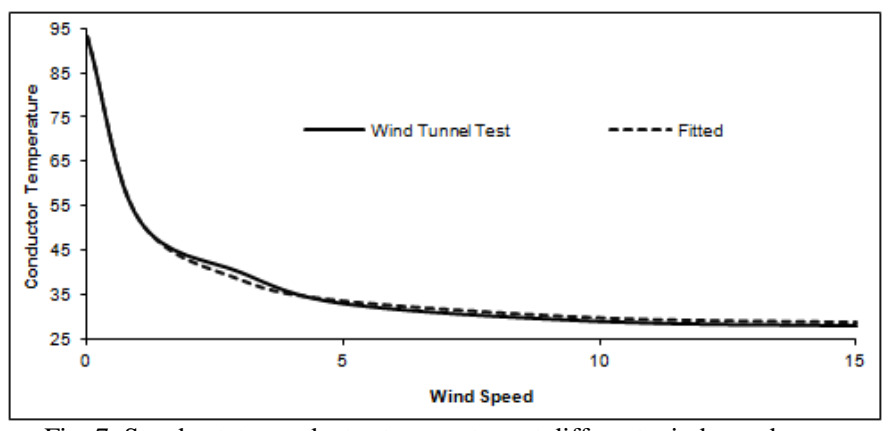

Fig. 7. Steady state conductor temperature at different wind speeds

TABLE I. THERMAL TIME CONSTANT FOR WIND TESTS

\begin{tabular}{|c|c|c|}
\hline \multirow{2}{*}{$\begin{array}{c}\text { Wind speed } \\
(\mathrm{m} / \mathrm{s})\end{array}$} & \multicolumn{2}{|c|}{ Time constants (minute) } \\
\cline { 2 - 3 } & Core & Surface \\
\hline 1 & 7.600 & 7.466 \\
\hline 3 & 4.125 & 4.155 \\
\hline 5 & 2.519 & 1.984 \\
\hline 10 & 1.567 & 1.561 \\
\hline 15 & 1.329 & 1.304 \\
\hline
\end{tabular}

\section{Field Measurements}

Field measurements have been carried out on two $110 \mathrm{kV}$ single circuits Omagh-Dungannon A $(36.1 \mathrm{~km})$ and B $(39.3 \mathrm{~km})$ since February 2008. The monitored lines pass over various terrains, including hills, valleys, forests, grassland, farmland etc. that the surrounding atmospheric conditions may vary from different sections of lines. So 
that the wind cooling effect on the conductor's temperature may be restricted by local sheltering factors. Moreover, both circuits are supported by wood pole portal structures varying from 13 to $20 \mathrm{~m}$ in height that result in experiencing weaker wind cooling compared to the higher tower structures. Under these conditions, the monitoring locations had to be chosen carefully to include the sections which are most likely to be overheated, i.e. critical spans, because the ampacity of the line is limited by the hottest sections. Thereupon, 10 locations as shown in Table II, mainly at low wind speed areas, have been selected as the on each circuit. Whereas, a few places with medium and high wind speed are also monitored for comparisons.

\section{TABLE II MONITORING LOCATION SELECTION}

\begin{tabular}{|l|l|l|l|}
\hline Line A & $\begin{array}{l}\text { Reason for } \\
\text { election }\end{array}$ & line B & Reason for selection \\
\hline A1 & $\begin{array}{l}\text { Substation terminal } \\
\text { tower }\end{array}$ & B1 & Termination of line \\
\hline $\begin{array}{l}\text { A2,3,4, } \\
7\end{array}$ & Low wind speed & $\begin{array}{l}\text { B2,3,6, } \\
8\end{array}$ & Low wind speed \\
\hline A5 & $\begin{array}{l}\text { Low wind speed, } \\
\text { river and small } \\
\text { forest }\end{array}$ & B4 & High wind speed \\
\hline A6 & $\begin{array}{l}\text { Medium wind } \\
\text { speed }\end{array}$ & B5 & Medium wind speed \\
\hline A8 & $\begin{array}{l}\text { Low wind speed } \\
\text { and sheltered by } \\
\text { trees }\end{array}$ & B7 & High wind speed \& trees \\
\hline A9 & $\begin{array}{l}\text { High wind speed } \\
\text { and high terrain }\end{array}$ & B9 & $\begin{array}{l}\text { Between road and river, } \\
\text { Medium wind speed }\end{array}$ \\
\hline A10 & $\begin{array}{l}\text { Medium wind } \\
\text { speed and road on } \\
\text { each side }\end{array}$ & B10 & $\begin{array}{l}\text { A \& B circuit close } \\
\text { together }\end{array}$ \\
\hline
\end{tabular}

At each measuring location, a set of monitoring devices are setup as shown in Fig 8. A Davis Vantage ${ }^{\mathrm{TM}}$ Pro II weather station is installed on the support structure at the same height of the overhead lines that ensures same atmospheric conditions are experienced. This device measures ambient temperature, instantaneous and average wind speed, wind direction and solar radiation with an archive interval of 5 minutes. While a line mounted device (FMC technologies) is employed for measuring conductor temperature and current on each of the three phases. Then, a data collection and transmission unit (Tnet) is mounted on the pole or tower at each site to receive the signals from the weather station and the FMC sensor, via an RS232 cable and an ISM (industrial, scientific and medical) band ratio signal respectively. Finally, all collected data are GPS stamped, and then send out using GPRS to a remote server, which can be accessed through the web controller.

\section{PLS Model For Thermal Behaviour Of The Conductor}

The model proposed in this work is a multivariate linear regression based PLS model. Although the PLS determines a linear relationship between the input and output parameters, the way it is applied in this work makes it able, to a good extent, to include non-linearity in input(weather and load parameters)/output(conductor temperature) relationship. Not only that, but also imitating the transient thermal behavior of the conductor.

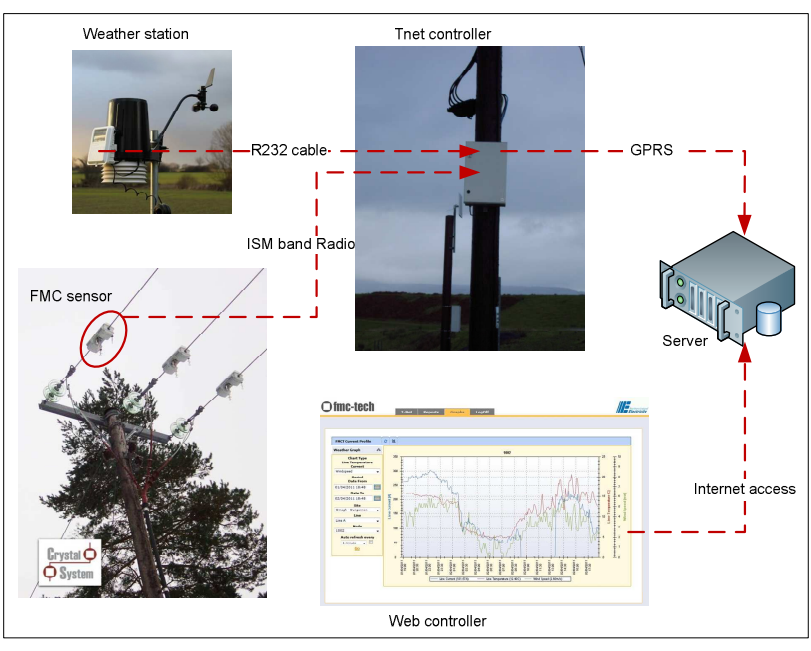

Fig. 8. Monitoring system for field measurements

Several online resources are available describing what is PLS and how it works. Therefore, details of PLS are not repeated here. All that we are interested in is to obtain a model that relates the conductor temperature to the current through the conductor as well as the weather parameters. This model is required to be simple and has no complicated calculations. It is essential for the model not to require the conductor physical parameters as these cannot be accurately knows and are not the same for all the line sections. It is also required that the model captures the effect of transient variations in weather parameters and conductor current on conductor temperature.

The PLS requires two distinct blocks of data. The first, $\mathbf{X}$ block, represents $m$ samples of $n$ independent (input) variables. The second, $\mathbf{Y}$ block, represents $m$ samples of $r$ dependent (output) variables. The output is the conductor temperature. So the $\mathbf{Y}$ block contains the temperature measurements. The input, $\mathbf{X}$ block, parameters are chosen as follows:

To account for transient thermal behavior of the conductor it was essential to take at least 3 consecutive measurements as mentioned before.

To consider the non-linearity, a quantities derived from direct measurements are used in addition to the measurements or instead of it. Square of the current measurements is used instead of the current. Also, terms derived from wind speed are used to reflect the nonlinear relationship between wind speed and conductor temperature as found by wind tunnel test and shown in Fig. 7.

To consider the wind speed normal to the conductor, terms of the form $V_{w} \cdot \sin (\theta)$ are used also as entries in the $\mathbf{X}$ block.

In case of one output variable, the PLS model yields a relationship of the form

$y=a_{0}+a_{1} x_{1}+a_{2} x_{2}+\cdots+a_{n} x_{n}$

The case of conductor temperature is a single output and the PLS model for it will yield a linear relationship similar to (1). The proposed PLS model for this case is as follows. 


$$
\begin{aligned}
T_{C}(t)=a_{0}+a_{1} * T_{a} & (t)+a_{2,3,4} e^{-b\left(V_{w}(t, t-1, t-2)\right)^{c}} \\
& +a_{5,6,7} V_{w}(t, t-1, t-2) e^{-b\left(V_{w}(t, t-1, t-2)\right)^{c}} \\
& +a_{8,9,10} V_{w} \sin (\theta)(t, t-1, t-2) \\
& +a_{11,12,13} S R(t, t-1, t-2) / 100+a_{14,15,16} \\
& * I^{2}(t, t-1, t-2) / 10^{5}
\end{aligned}
$$

Where: $T_{c}$ is the conductor temperature, $T_{a}$ is the ambient temperature, $V_{w}$ is the wind speed, $S R$ is the solar radiation, and $I$ is the conductor current. The notation $\mathrm{x}(\mathrm{t}, \mathrm{t}-1, \mathrm{t}-2)$ means three terms at the three consecutive instances $\mathrm{t}-2, \mathrm{t}-1$, and $\mathrm{t}$.

The model defined by (2) is the general form of 6 different models. The six models differ from each other by the terms of (2) which are considered by each model. Field measurements have been used for determining the coefficients of the different models at one measurement location. Table III lists the coefficients of the six models. If a coefficient is set to zero means that the corresponding term is excluded from the model; that is to say that model 1 has all the terms of (2) considered, while in model 2 the $V_{w}(t-2)$ is excluded. The last two rows of Table III displays the average error and the standard deviation of the error for each model. It can be noticed that the average error for all the six models is almost the same with very little difference between them. It can also be noticed that the lowest error is that of M1, the model with the largest number of terms. However, for $\mathrm{M} 1$, the conductor temperature sensitivity to $V_{w} \sin (\theta)(t-2)$ is positive. It is to be observed that the total sensitivity of conductor temperature to the wind speed components, the sum of $a_{8^{-}}$ 10 , is almost the same as for all the models including M1. However, the positive sensitivity to one component is not that acceptable. Therefore, this component was removed from all the other models.

As stated above, the difference between the 6 models is not that significant and this introduces some freedom, arbitrariness, in the choice of the model. However, it is expected that this freedom or arbitrariness would disappear when the line gets heavily loaded and the conductor temperature gets near to maximum.

To test the accuracy of the proposed PLS model the conductor temperature calculated by the PLS model, M6, is compared to the measured conductor temperature. The model was trained with the measurements of March 2009. Both the measured and estimated conductor temperature for a sample day of March 2009 are plotted in Fig. 9.a, which shows the accuracy of the PLS model in calculating the conductor temperature very close to the measured one. Fig. 9.b shows the same but for a sample day of April 2009, which has not been used in training the PLS model. The model is also accurate but with a slightly larger error, especially in the low temperature periods.

Fig. 10 shows a scatter plot of the absolute error in conductor temperature prediction against time of the day for the whole month. It is clear that except for relatively number of measurements the error is less than one degree. It can be noticed that the few points of higher error are located around midday due to the effect of short cloudy periods on the incident solar radiation.
TABLE III PLS MODEL COEFFICIENTS FOR 6 DIFFERENT MODELS

\begin{tabular}{|c|c|c|c|c|c|c|}
\hline \multirow{2}{*}{ Coef } & \multicolumn{7}{|c|}{ Models } \\
\cline { 2 - 7 } & $\mathrm{M} 1$ & $\mathrm{M} 2$ & $\mathrm{M} 3$ & $\mathrm{M} 4$ & $\mathrm{M} 5$ & M6 \\
\hline$a_{0}$ & 0.1533 & 0.1883 & 0.6033 & 0.5986 & 0.5986 & 0.6020 \\
\hline$a_{1}$ & 1.0678 & 1.0681 & 1.0683 & 1.0684 & 1.0684 & 1.0673 \\
\hline$a_{2}$ & 0.0101 & 0.0024 & -.0497 & 0 & 0 & 0 \\
\hline$a_{3}$ & 0.0496 & 0.02152 & -.3599 & -.4032 & -.4034 & -.3972 \\
\hline$a_{4}$ & 0.3817 & 0.6093 & 0 & 0 & 0 & 0 \\
\hline$a_{5}$ & -.0033 & -.0065 & -.0107 & -.0065 & 0 & 0 \\
\hline$a_{6}$ & -.0858 & -.1102 & -.1168 & -.1205 & -.1267 & -.1288 \\
\hline$a_{7}$ & -.0433 & -.0404 & -.0395 & -.0394 & -.0399 & 0 \\
\hline$a_{8}$ & -.0403 & -.0377 & -.0368 & -.0368 & -.0373 & -.0544 \\
\hline$a_{9}$ & -.0871 & -.0385 & -.0377 & -.0376 & -.0371 & -.0489 \\
\hline$a_{10}$ & 0.0521 & 0 & 0 & 0 & 0 & 0 \\
\hline$a_{11}$ & 0.4206 & 0.4204 & 0.4198 & 0.4198 & 0.4200 & 0.4216 \\
\hline$a_{12}$ & 0.3925 & 0.3926 & 0.3924 & 0.3923 & 0.3923 & 0.3912 \\
\hline$a_{13}$ & 0.2399 & 0.2393 & 0.2393 & 0.2391 & 0.2390 & 0.2377 \\
\hline$a_{14}$ & 0.1608 & 0.1580 & 0.1551 & 0.1546 & 0.1545 & 0.1479 \\
\hline$a_{15}$ & 0.0673 & 0.0681 & 0.0683 & 0.0684 & 0.0683 & 0.0752 \\
\hline$a_{16}$ & 0.0747 & 0.0707 & 0.0690 & 0.0693 & 0.0694 & 0.0624 \\
\hline Av. Err. & 0.5328 & 0.5353 & 0.5368 & 0.5369 & 0.5368 & 0.5376 \\
\hline St. Dev. & 0.4975 & 0.4963 & 0.4951 & 0.4949 & 0.4950 & 0.4960 \\
\hline
\end{tabular}

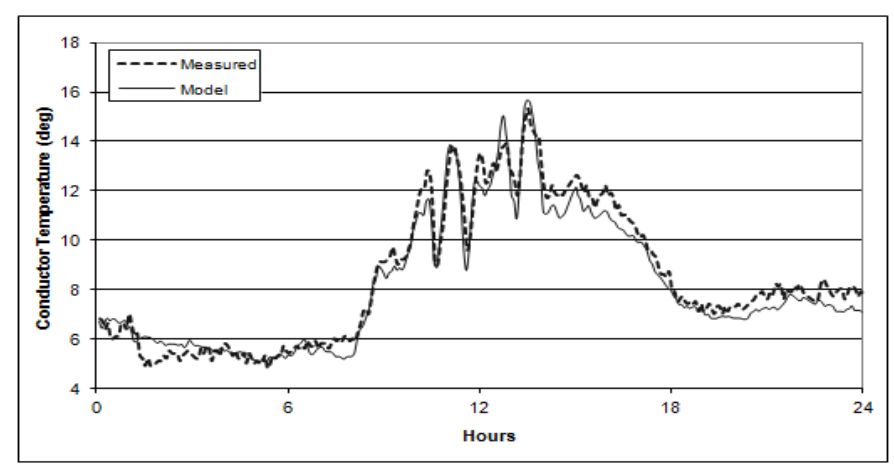

a. Test data of the same month used in PLS training

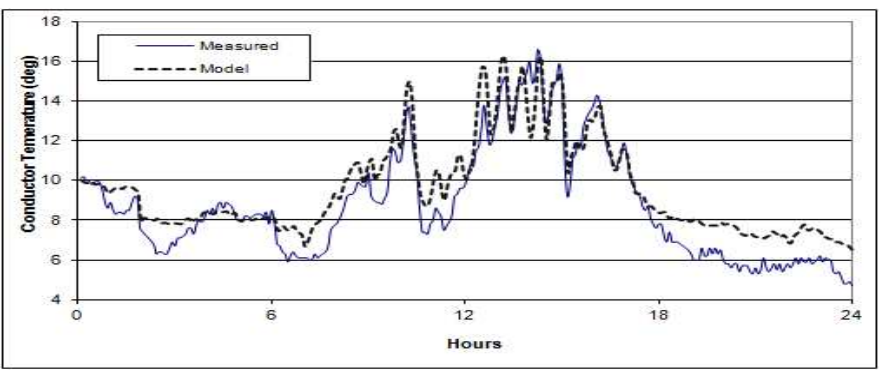

b. Test data from a month other than that used in PLS training

Fig. 9 Comparison of measured and estimated conductor temperature

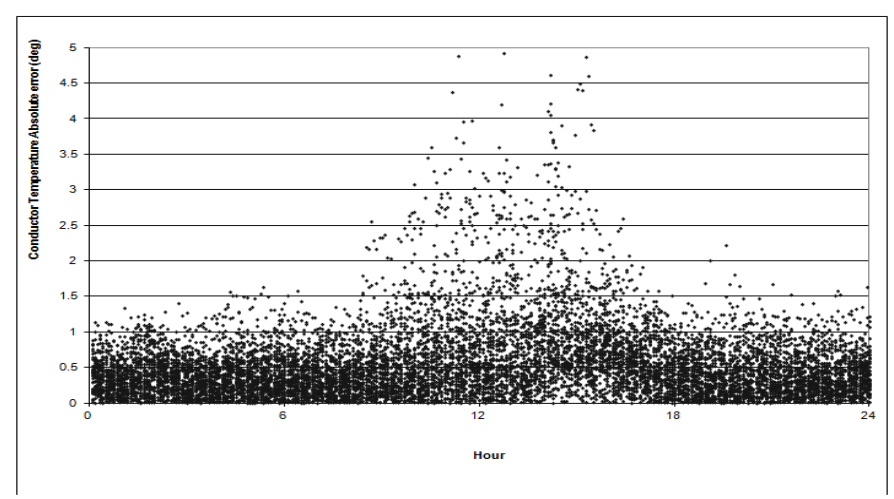

Fig. 10 Estimated temperature error against time of the day 


\section{Conclusion}

The paper presents a model for conductor temperature as a function of line current and weather parameters. The proposed model does not require knowledge of the line parameters such as resistivity and emissivity as it is based on measurements. The conductor temperature is represented as a linear function of weather parameters, line current and some quantities derived from both. Decision about the model structure, the quantities used, the number of observations used in one calculation step and the measurement period has been made based on the results of lab tests.

Lab tests, both still air and wind tunnel are also presented with the results that were used to decide about the model structure.

The parameters of different models have been determined for a sample month of the measured data and presented along with a comparison of the measured and the estimated conductor temperature, both for the sample month used for determining the model and another month. The model shows a good accuracy in determining the conductor temperature with an average error of the order of 0.5 degree.

The linearity of the proposed model makes it simple to use and enable the determination of a day, or whatever period, ahead dynamic line rating forecast. This is very important to the power system operator/operation planner as it enables to have a realistic estimate of the available transmission capacity rather than the traditional static rating based on the assumption of worst-case conditions. It also helps to accommodate more power from the wind as it is most likely that line rating is higher during windy periods.

The accuracy of the model would be greatly improved when high current and high conductor temperature are available. A test rig is now installed at QUB to enable testing the conductor at real weather conditions with high current and at high conductor temperature. The purpose of which is to get the required measurements and also to validate the model. The results will be reported soon when it is complete.

\section{Acknowledgment}

This work was supported in part by Science Foundation Ireland (SFI) through the Charles Parsons Energy Research Award: 06/CP/E002.

\section{References}

[1] I. T and D. Committee, "IEEE standard for calculating the currenttemperature of bare overhead conductors," IEEE Standard 738, 2007.

[2] W. G. CIGRE, "Thermal Behaviour of Overhead Conductors," 2002

[3] B. Howington and G. Ramon, "Dynamic thermal line rating summary and status of the state-of-the-art technology," Power Delivery, IEEE Transactions on, vol. 2, no. 3, pp. 851-858, 1987.

[4] S. D. Foss and R. Maraio, "Dynamic line rating in the operating environment," Power Delivery, IEEE Transactions on, vol. 5, no. 2, pp. 1095-1105, 1990.

[5] D. A. Douglass, D. C. Lawry, A. A. Edris, and E. C. Bascom III, "Dynamic thermal ratings realize circuit load limits," Computer Applications in Power, IEEE, vol. 13, no. 1, pp. 38-44, 2000.

[6] P. M. Callahan and D. A. Douglass, "An experimental evaluation of a thermal line uprating by conductor temperature and weather monitoring”, IEEE Transactions on Power Delivery, Vol. 3, No. 4, 1988, 1960-1967.

[7] J. Engelhardt and S. Basu, "Design, installation, and field experience with an overhead transmission dynamic line rating system," Transmission and Distribution Conference, 1996. Proceedings., 1996 IEEE, pp. 366-370, 1996.

[8] M. Berende, J. Slootweg, and G. Clemens, "Incorporating weather statistics in determining overhead line ampacity," Future Power Systems, 2005 International Conference on, p. 8-pp, 2005.

[9] M. M. Saied, "Assessing the dynamic rating of overhead transmission lines," European Transactions on Electrical Power, vol. 17, no. 5, pp. 526-536, 2007.

[10]J. Hosek, P. Musilek, E. Lozowski, and P. Pytlak, "Effect of time resolution of meteorological inputs on dynamic thermal rating calculations," Generation, Transmission \& Distribution, IET, vol. 5, no. 9, pp. 941-947, 2011.

[11]M. Matus, D. Saez, M. Favley, C. Suazo-Martinez, J. Moya, G. Jimenez-Estevez, R. Palma-Behnke, G. Olguin, and P. Jorquera, "Identification of Critical Spans for Monitoring Systems in Dynamic Thermal Rating", IEEE Transactions on Power Delivery, Vol. 27, No. 2, 2012, pp. 1002-1009.

[12]C. Mensah-Bonsu, U. F. Krekeler, G. T. Heydt, Y. Hoverson, J. Schilleci and B. L. Agrawal, "Application of the Global Positioning System to the measurement of overhead power transmission conductor sag", IEEE Transactions on Power Delivery, Vol. 17, No. 1, 2002, pp. 273-278.

[13]M. RuiKun, F. Ling and X. HaiBo, "Dynamic Line Rating estimator with synchronized phasor measurement", International Conference on Advanced Power System Automation and Protection (APAP), 2011, pp. 940-945.

[14]S. Abbot, "Investigation into the effect of wind cooling on overhead lines”, A graduation project, Queen's University Belfast, 2008. 